\title{
Publish or Perish?
}

\author{
Kostas Kampourakis ${ }^{1}$
}

Published online: 1 April 2016

(C) Springer Science+Business Media Dordrecht 2016

Being the editor-in-chief of a journal like Science \& Education has many interesting aspects. One, perhaps the most important, is the creative aspect. Published articles are primarily the product of the work of their authors, and so they are the most creative of all. But published articles are also the product of intellectual input from reviewers, which-I can testify from experience - can lead to significant improvements. As already discussed in a previous editorial, the editorial team of Science \& Education considers peer review to be an important process for strengthening one's work, and not only as a sorting process. Thus, reviewers contribute to the improvement of submitted manuscripts by illuminating unseen conceptual connections, introducing valuable references, and by identifying hidden weaknesses. For these reasons, both authors and reviewers can be viewed as creative and scholarly partners. What about editorial work? Can creative contributions be found there?

Indeed, they can. Whenever we receive a manuscript, we read it carefully and work to identify a diverse set of expert reviewers. There are several considerations to make: (1) the reviewers must be qualified and be able to make valuable suggestions that will help the authors improve their work; (2) in the case of our journal, reviewers have to come from different disciplines: education, philosophy, history, sociology of science or mathematics, and so we must find an appropriate combination of reviewers that can collectively cover the topic being investigated; and (3) the reviewers must encompass a range of viewsparticularly on hotly debated topics - to ensure a balanced perspective. Reviewer selection therefore emerges as an important creative aspect of the editorial process.

But there are certain preconditions for such creative work, the most important ones being manuscript quality and appropriateness for Science \& Education. Unfortunately, I devote large amounts of time reading low-quality manuscripts and/or very poorly suited to the journal. One reason for this problem is the push to publish ever-increasing numbers of papers in order to meet promotion and funding requirements. As a result, authors seem to rush to submit manuscripts without ensuring that they have been clearly written and

Kostas Kampourakis

Kostas.Kampourakis@unige.ch

1 University of Geneva, Pavillon Mail, 40 Boulevard du Pont-d'Arve, 1211 Geneva, Switzerland 
carefully checked. Unfortunately, it can be the case that strong work may be hidden behind a poorly written text. Indeed, even if we see some potential in a manuscript, we often have to reject it without review because of the enormous time and effort that would be required to make it ready for journal reviewers.

In some cases, we receive interesting manuscripts that lack the crucial HPS dimension, which is essential for work to be forwarded to reviewers. We are often disappointed to have to send promising studies back to authors because they lack this dimension. Too much time and effort is wasted-for authors and for editors. Worse than that, in other cases authors submit manuscripts that clearly fall outside the scope of our journal. These are manuscripts that often have nothing to do with science education at all. It must be clear that the journal is interdisciplinary and thus authors from different disciplines can contribute for it. But a manuscript written, e.g. by a historian, a philosopher or a scientist should have explicit implications for teaching and learning. There is a reason that the term "Education" is part of the title of the journal.

Why is this happening? Because in many cases in order to get a job, a promotion, or a research grant, one has to submit a list of publications. So, the more publications one has, the more likely one is to get the job or the funding. If one does not have an adequate number of publications, whatever that number is, there is no job, no promotion and no funding. This is what the phrase "publish or perish" encapsulates. Either you publish like crazy or you are out of the competition. But is publishing all that matters? What about teaching? Does teaching matter as much as publications? In many cases, of course it does, but I am aware of several other cases where it makes no difference at all. Yet, researchers holding professorial posts in universities are supposed to both do research and teach. Teaching experience may be irrelevant in order to get a research grant, and in such cases previous research experience and published work should matter. But when someone is hired to teach and do research, why privilege one over the other?

In my view, a valuable approach that scholars could adopt is to submit two equally important lists: a list of publications and a list of the courses taught in the past-along with their evaluations. If that were always the case, people would also be rushing to teach well, and not only to publish in peer-reviewed journals. What can be done about this? Apparently, the first step would be to consider both the teaching record and the publications list as essential and equally important criteria for hiring a professor. Of course, this is already the case in several universities, but it is not the case everywhere. Then, there is a second step that we should follow: help graduate students in any discipline understand that they must continuously work to improve the quality of their teaching as well as conduct and publish research. If this happened everywhere, then the next generation of professors might make the first step that the current generation may be reluctant to make.

In this way, the journal-publishing process could become a more creative one. Without the rush to publish, scholars could diligently prepare their manuscripts and then submit to the appropriate journals, not to any journal. Any publication should not count as better than no publication. Publications should be the means to spread one's research, thoughts, ideas and arguments, in order to inform and educate others, not to get promotion and funding. So, let us all hope that we can enhance the creative aspect of this process. Let us all publish for the sake of publishing itself, for education, and not for factors relevant but external to it. 